The AstrophysiCAL JouRNAL, 525:1032-1041, 1999 November 10

(c) 1999. The American Astronomical Society. All rights reserved. Printed in U.S.A.

\title{
STANDARD SOLAR MODELS IN THE LIGHT OF NEW HELIOSEISMIC CONSTRAINTS. II. MIXING BELOW THE CONVECTIVE ZONE
}

\author{
A. S. BRUN, ${ }^{1,2}$ S. TURCK-ChIÈZE ${ }^{1}$ AND J. P. ZAHN ${ }^{2}$ \\ Received 1999 March 1; accepted 1999 July 2
}

\begin{abstract}
In previous work, we have shown that recent updated standard solar models cannot reproduce the radial profile of the sound speed at the base of the convective zone and fail to predict the photospheric lithium abundance. In parallel, helioseismology has shown that the transition from differential rotation in the convective zone to almost uniform rotation in the radiative solar interior occurs in a shallow layer called the tachocline. This layer is presumably the seat of a large-scale circulation and of turbulent motions. Here we introduce a macroscopic transport term in the structure equations that is based on a hydrodynamical description of the tachocline proposed by Spiegel \& Zahn, and we calculate the mixing induced within this layer. We discuss the influence of different parameters that represent the tachocline thickness, the Brunt-Väisälä frequency at the base of the convective zone, and the time dependence of this mixing process along the Sun's evolution. We show that the introduction of such a process inhibits the microscopic diffusion by about $25 \%$. Starting from models including a pre-main-sequence evolution, we obtain (1) a good agreement with observed photospheric chemical abundance of light elements such as ${ }^{3} \mathrm{He},{ }^{4} \mathrm{He},{ }^{7} \mathrm{Li}$, and ${ }^{9} \mathrm{Be}$; (2) a smooth composition gradient at the base of the convective zone; and (3) a significant improvement of the sound-speed square difference between the seismic Sun and the models in this transition region when we allow the photospheric heavy-element abundance to adjust, within the observational incertitude, as a result of the action of this mixing process. The impact on neutrino predictions is also discussed.
\end{abstract}

Subject headings: diffusion - Sun: abundances - Sun: interior - Sun: oscillations

\section{INTRODUCTION}

The validation of solar structure is crucial for checking the hypotheses used in stellar evolution modeling. It allows also the helium content at the birth of the Sun to be determined, which is key to understanding Galactic enrichment and for predicting the present neutrino emission fluxes. With the support of helioseismology, we are now able to constrain the sophisticated calculations performed to describe the present structure of the Sun. Very recently, a tremendous effort using ground-based networks and space experiments has been made to improve the accuracy of the seismic tool at a level where differences with the theoretical estimates are significant. Consequently, it has been demonstrated that this discipline has the potential to check classical stellar structure for solar-type stars in the hydrogen-burning phase (Tomczyk et al. 1995; Kosovishev et al. 1997; Turck-Chièze et al. 1997; ChristensenDalsgaard 1998).

In parallel, the development of neutrino detectors in particle physics has the objective of determining the properties of these particles. In this research, the Sun plays, once again, a specific role as a source of electronic neutrinos $v_{e}$ that could be transformed into other flavors before reaching the detectors on Earth (Hata \& Langacker 1997; Bahcall, Krastev \& Smirnov 1998). Actual solar conditions are almost impossible to reproduce in experiments on Earth, so one must predict precisely the emitted neutrino fluxes in order to progress in our knowledge of these particles and the solar core.

At first glance, helioseismology shows that the standard solar framework is correct (Brun, Turck-Chièze, \& Morel

\footnotetext{
${ }^{1}$ DSM/DAPNIA/Service d'Astrophysique, CEA Saclay, 91191 Gif-surYvette, France.

${ }^{2}$ Département d'Astrophysique Stellaire et Galactique, Observatoire de Paris, Section Meudon, 92195 Meudon, France.
}

1998, hereafter Paper I). It is nevertheless extremely clear that standard models have always failed to answer some well-known questions or anomalies because of their simplified assumptions (Turck-Chièze et al. 1993). These open questions concern the effects of the rotation and magnetic field, the history of the angular momentum, the actual way the convection acts, the unexplained photospheric abundances as, for example, the photospheric lithium depletion, and the presence of mixing at different locations inside the stars. Recent results obtained with the $\mathrm{SOHO}$ satellite (Fröhlich et al. 1997; Kosovishev et al. 1997; Gabriel et al. 1997) and ground networks have demonstrated that there exist significant differences in the square of the sound speed between the Sun and the models (Kosovishev et al. 1997; Turck-Chièze et al. 1997). Some of them can be attributed to uncertainties in the microscopic description of the Sun, while others must be signs of processes missing in the standard solar framework.

The aim of this paper is to introduce a new term in the stellar structure equations in order to progress beyond the standard framework. This new term describes some physical processes that are presumably present in our star, as suggested by the differential rotation profile. In this study, we hope to treat more correctly the transition layer between the convective and radiative zones with the help of crucial measured helioseismic variables. The main reasons for our interest in this layer are the following:

1. The change of rotation rate (solid $\Rightarrow$ differential) measured by helioseismology (Thompson et al. 1996; Kosovichev et al. 1997) probably develops some turbulent mixing in a shallow layer (tachocline) that could be where the magnetic field is anchored and amplified by a dynamo process (Schüssler 1987; Choudhuri, Schüssler, \& Dikpati 1997).

2. The standard solar model framework, which includes microscopic diffusion and element settling, has failed to 
treat this transition correctly (Paper I). Such a model leads to sharp element profiles, which create a large bump in the sound-speed profile below the convective zone and cannot reproduce the observed lithium depletion

3. The turbulent convective zone surely interacts with the stably stratified radiative zone (e.g., the Brunt-Väisälä frequency $N^{2}>0$ ), developing turbulence, modifying the thermal structure of the star (convective penetration; Zahn 1991), extending the mixing zone (overshooting; Roxburgh 1997; Zahn 1998), or generating internal waves (Press 1981; Schatzman 1993).

Following the description of Spiegel \& Zahn (1992, hereafter SZ92) of the motions in this so-called tachocline and that of Chaboyer \& Zahn (1992) for the chemical evolution, we introduce an effective diffusion coefficient into the equations of the stellar structure and build several solar models which are compared to the helioseismic and surface abundance observations.

We first present in $\S 2$ the physical model of the solar tachocline we use and deduce the vertical macroscopic diffusion coefficient to be introduced in the time-evolution equation of the chemical abundances. Then, in $\S 3$, we present solar models with a mixing occurring in the tachocline and discuss the effect of the time evolution of such a mixing from the Sun's early phases until the present day. Finally, in $\S 4$ we discuss our results, and we conclude in $\S 5$.

\section{THE SOLAR TACHOCLINE}

The Sun is generally assumed to be in hydrostatic and thermal equilibrium, neglecting the effects of rotation and magnetic field. However, models built with these simplifying assumptions do not totally agree with the helioseismic data, in particular with those obtained by $\mathrm{SOHO}$. It appears that macroscopic mixing processes must be taken into account not only in the convective zone but also in the radiative interior. Macroscopic mixing may be introduced in the solar models by adding an effective diffusivity $D_{T}$ in the equation governing the time evolution of the chemical species $X_{i}$. The equation becomes:

$$
\frac{\partial X_{i}}{\partial t}=-\frac{\partial\left(4 \pi \rho r^{2} X_{i} V_{i}\right)}{\partial m}+\text { nuclear terms, }
$$

where the velocity $V_{i}$ of species $i$ with respect to the center of mass is

$$
V_{i}=-4 \pi \rho r^{2}\left(D_{i}+D_{T}\right) \frac{\partial \ln X_{i}}{\partial m}+v_{i} .
$$

The velocity $V_{i}$ is the sum of a term that depends on the concentration gradient and another that does not, $v_{i}$ (Burgers 1969; Proffitt \& Michaud 1991). In this paper, we add a macroscopic diffusion term $D_{T}$ to the microscopic diffusion term $D_{i}$. This will describe the mixing occurring in the shear layer connecting the differential rotation in the convection zone to the solid rotation in the radiative interior. As presented in SZ92, a possible physical interpretation of this tachocline is obtained by invoking anisotropic turbulence with much stronger viscous transport in the horizontal than in the vertical direction (i.e., $v_{H} \gg v_{V}$ ). Such turbulence reduces the differential rotation and therefore inhibits the spread of the layer deep inside the radiative zone, leading to a stationary state. For a justification of this anisotropy, we refer to Michaud \& Zahn (1998).

\subsection{The Hydrodynamical Description}

The conservation of mass, momentum, and entropy is given by

$$
\begin{gathered}
\frac{\partial \rho}{\partial t}+\nabla \cdot(\rho \boldsymbol{V})=0 \\
\rho\left[\frac{\partial \boldsymbol{V}}{\partial t}+(\boldsymbol{V} \cdot \boldsymbol{\nabla}) \boldsymbol{V}+2 \boldsymbol{\Omega} \wedge \boldsymbol{V}+\dot{\boldsymbol{\Omega}} \wedge \boldsymbol{r}\right] \\
=-\nabla P-\rho \boldsymbol{\nabla} \Phi+\boldsymbol{\nabla} \cdot\|\tau\|, \\
\rho T\left(\frac{\partial}{\partial t}+\boldsymbol{V} \cdot \boldsymbol{\nabla}\right) S=\boldsymbol{\nabla} \cdot(\chi \nabla T),
\end{gathered}
$$

where $\rho$ is the density, $P$ is the pressure, $T$ is the temperature, and $\boldsymbol{V}=(u, v, r \hat{\Omega} \sin \theta)$ is the local velocity in the rotating frame $\boldsymbol{\Omega}$. In this expression, the differential rotation $\hat{\Omega}$ appears explicitly, $S$ is the specific entropy, $\|\tau\|$ the viscous stress tensor, and is $\Phi$ the gravitational potential. In order to ease the solution of this system of equations, some simplifying assumptions were made by SZ92, mainly,

1. Velocities are small compared to the sound speed: $\partial \rho / \partial t$ is negligible (anelastic approximation).

2. Advection terms and viscous forces are small compared to the Coriolis acceleration (i.e., small Rossby and Ekman numbers).

3. The tachocline is thin compared to the pressure scale height, and the turbulent horizontal viscosity is stronger than the vertical one (i.e., $v_{H} \gg v_{V}$ ).

After separating each variable into its mean value on the sphere plus a perturbation, e.g., $T(r, t)+\hat{T}(r, \theta, t)$, SZ92 give the linearized form of the equations in a stationary state. Spiegel \& Zahn define a stream function $\Psi$ for the meridional flows by

$$
r^{2} \rho u=\frac{\partial \Psi}{\partial x}, \quad r \rho v \sin \theta=\frac{\partial \Psi}{\partial r},
$$

where $x=\cos \theta$, and project the variables of the equations describing the tachocline on horizontal eigenfunctions $F_{i}$ :

$$
\begin{aligned}
(\hat{P}, \hat{T}, u) & =\sum_{i}\left(\tilde{P}_{i}, \widetilde{T}_{i}, u_{i}\right) F_{i}(x), \\
\Psi & =\sum_{i} \tilde{\Psi}_{i} \int F_{i}(x) d x, \\
x \hat{\Omega} & =\sum_{i} \widetilde{\Omega}_{i} \frac{d F_{i}}{d x} .
\end{aligned}
$$

The dominant term for the even eigenfunctions is $i=4$, but we will consider in our calculation even terms up to $i=8$. From this projection, SZ92 deduce a fourth-order differential equation for the modal function $\widetilde{\Omega}_{i}(r)$, defined in equation (7):

$$
\begin{aligned}
\frac{4 \Omega^{2}}{\mu_{i}^{4}} \frac{1}{\rho v_{H}} \frac{\partial}{\partial r}\left\{\frac{g}{N^{2} c_{p} T} \frac{\partial}{\partial r}\left[\chi r^{2} \frac{\partial}{\partial r}\left(\frac{T}{\rho g} \frac{\partial}{\partial r} \rho r^{2} \widetilde{\Omega}_{i}\right)\right]\right\} & +\widetilde{\Omega}_{i} \\
& =0,
\end{aligned}
$$

where $\mu_{i}$ are eigenvalues related to the eigenfunctions $F_{i}$ (for $\left.i=4, \mu_{4}=4.933\right), \chi=16 \sigma T^{3} / 3 \kappa \rho$ is the radiative conductivity, $N$ is the Brunt-Väisälä frequency, $\Omega$ is the mean rota- 
tion rate of the radiation zone, and all the other variables have their usual meaning.

The problem is simplified by treating the tachocline as a boundary layer in which the rapidly varying quantity is $\tilde{\Omega}_{i}$ and all the other physical quantities are assumed to be constant. By doing so, an analytical solution of the simplified fourth-order differential equation is (for simplicity we give only the expression for the modal index $i=4$ )

$$
\tilde{\Omega}_{4}(\zeta)=\widetilde{\Omega}_{4}(0) \sqrt{2} \exp (-\zeta) \cos (\zeta-\pi / 4),
$$

where $\widetilde{\Omega}_{i}(0)=Q_{i} \Omega, Q_{i}$ are numerical coefficients depending on the latitudinal variation of the differential rotation, and we have defined a nondimensional depth,

$$
\zeta=\mu_{i}\left(r_{\mathrm{bcz}}-r\right) / d,
$$

with

$$
d=r_{\mathrm{bcz}}(2 \Omega / N)^{1 / 2}\left(4 K / v_{H}\right)^{1 / 4}
$$

being a parameter related to the tachocline thickness $h, r_{\mathrm{bcz}}$ greater than the radius, and $K=\chi / \rho c_{p}$, the radiative diffusivity at the base of the convective zone.

With the latitudinal dependence of the angular velocity at the base of the convection zone borrowed from Thompson et al. (1996), $\Omega_{\mathrm{bcz}}>/ 2 \pi=456-72 x^{2}-42 x^{4} \mathrm{nHz}$, we have reestimated the coefficient $Q_{4}=-1.707 \times 10^{-2}$ as well as the ratio between the rotation rate of the radiative zone and the equatorial rate, $\Omega / \Omega_{0}=0.9104$. The prediction by Gough \& Sekii (1997), who consider instead the magnetic stresses, is $\sim 0.96$; presently, the seismic observations suggest a rotational ratio of $0.94 \pm 0.01$ (Corbard et al. 1999), which is intermediate between these two theoretical estimates.

The inversion of the rotational profile from helioseismology observations gives the width of the tachocline and its location below the convection zone. For a comparison between the observational thickness and the theoretical one, we have to consider the first zero of equation (9), e.g., $h=3 \pi d / 4 \mu_{4} \sim d / 2$. The parameter $d$ is then approximately equal to $2 h$. In the solar models of $\S 3$, we will treat $h$ (and hence $d$ ) as an adjustable parameter in order to agree with the helioseismic inversion of the tachocline thickness (Basu 1997; Charbonneau, Christensen-Dalsgaard, \& Henning 1998; Corbard et al. 1998, 1999).

Our purpose now is to obtain an analytical expression for the vertical velocity $u$. We already have an expression for the differential rotation; the next step is to connect $u$ to $\hat{\Omega}$. From the conservation of the angular momentum, we find a relation between the stream function $\hat{\Psi}$ and the differential rotation $\hat{\Omega}$. By writing the stream function as $\widetilde{\Psi}_{i}=\rho r^{2} u_{i}$ and introducing the analytical expression of $\widetilde{\Omega}_{i}$ (eq. [9]), we finally obtain, after integration, the radial dependence of $u_{i}$; in the case of $i=4$ we get

$$
u_{4}(\zeta)=\frac{1}{2} \frac{v_{H} d}{r_{\mathrm{bcz}}^{2}} \mu_{4}^{3} Q_{4} \exp (-\zeta) \cos (\zeta)
$$

From equation (12) we shall derive an expression for the macroscopic diffusivity $D_{T}$ of the motions occurring in the tachocline.

\subsection{Macroscopic Diffusion of Chemical Species}

We determine in this section the influence of the mixing on the evolution of chemical species, using the expression for the vertical velocity amplitude $u_{i}$ (eq. [12]). The anisotropic diffusion, invoked by SZ92 to stop the spread of the tachocline deeper in the radiative zone, interferes with the advective transport of chemicals. Chaboyer \& Zahn (1992) have shown that the result is a diffusive transport in the vertical direction, with an effective diffusivity given by

$$
D_{T}=\frac{r^{2}}{D_{H}} \sum_{n} \frac{U_{n}^{2}(r)}{n(n+1)(2 n+1)},
$$

where $U_{n}$ are the coefficients of the expansion of the vertical component of the velocity $u$ in Legendre polynomials. The eigenfunctions $F_{i}$ introduced in $\S 2.1$ may be projected on these Legendre polynomials, and to a good approximation one has (keeping only the $n=4,6$, and 8 terms)

$$
D_{T}=\frac{r^{2}}{D_{H}}\left[\left(\frac{8}{3}\right)^{2} \frac{u_{4}^{2}(r)}{180}+\left(\frac{16}{5}\right)^{2} \frac{u_{6}^{2}(r)}{546}+\left(\frac{128}{35}\right)^{2} \frac{u_{8}^{2}(r)}{1224}\right] .
$$

One reaches the following expression for the vertical diffusivity by replacing $u_{4}$ in equation (14) by equation (12) and, similarly, $n=6$ and $n=8$ (see Fig. 1):

$$
\begin{aligned}
D_{T}(\zeta)= & \frac{1}{180} \frac{1}{4}\left(\frac{8}{3}\right)^{2} v_{H}\left(\frac{d}{r_{\mathrm{bcz}}}\right)^{2} \mu_{4}^{6} Q_{4}^{2} \exp (-2 \zeta) \\
& \times \cos ^{2}(\zeta)+\text { higher order terms, }
\end{aligned}
$$

where $\zeta$ is the scaled vertical coordinate defined in equation (10).

In this expression, it is assumed that the horizontal component of the macroscopic diffusivity $D_{H}$ is equal to the horizontal viscosity $v_{H}$. This coefficient is then introduced in the diffusive part of the equation (1) for the time evolution of the chemical abundances $X_{i}$, and we could thus obtain solar models including a treatment of the tachocline. Figure 1 compares the different values of the macroscopic diffusivity for models discussed in the next section to the microscopic diffusion (here represented as a horizontal solid
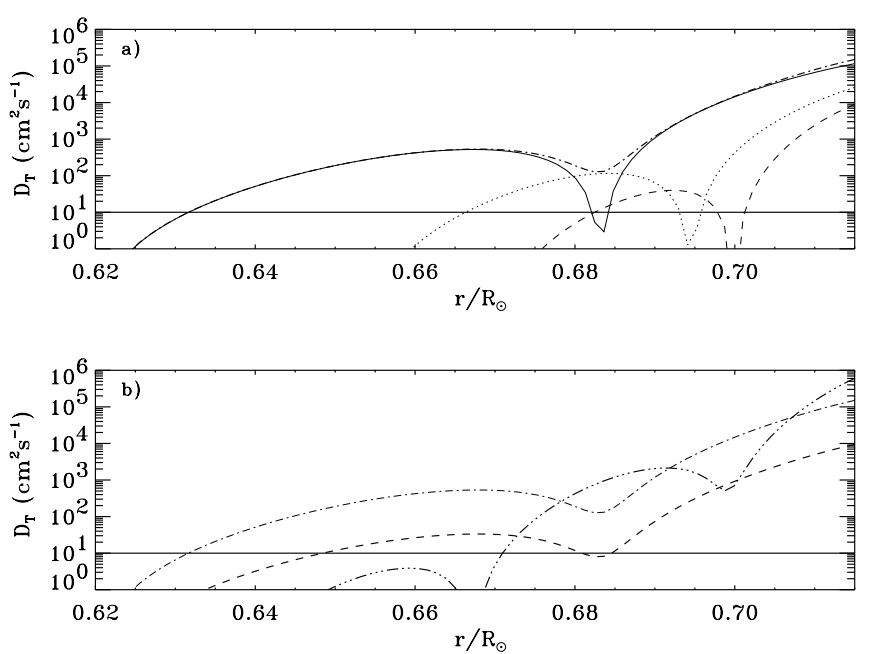

FIG. 1.-Effective diffusivity $D_{T}$ for tachocline mixing: (a) Model $B$ $(N=25$ and $d=0.1)$ for index $n=4 B_{4}$ (solid line), $n=6 B_{6}$ (dotted line), $n=8 B_{8}$ (dashed line) and $\sum_{n=4}^{8} B_{n}$ (thick dash-dotted line). (b) Models $A$ $(N=100$ and $d=0.1)$ (dashed line), $B(N=25$ and $d=0.1)$ (dash-dotted line) and $C(N=25$ and $d=0.5)$ (dash-triple-dotted line). The horizontal solid line represents approximately the microscopic diffusion $D_{i}$. 


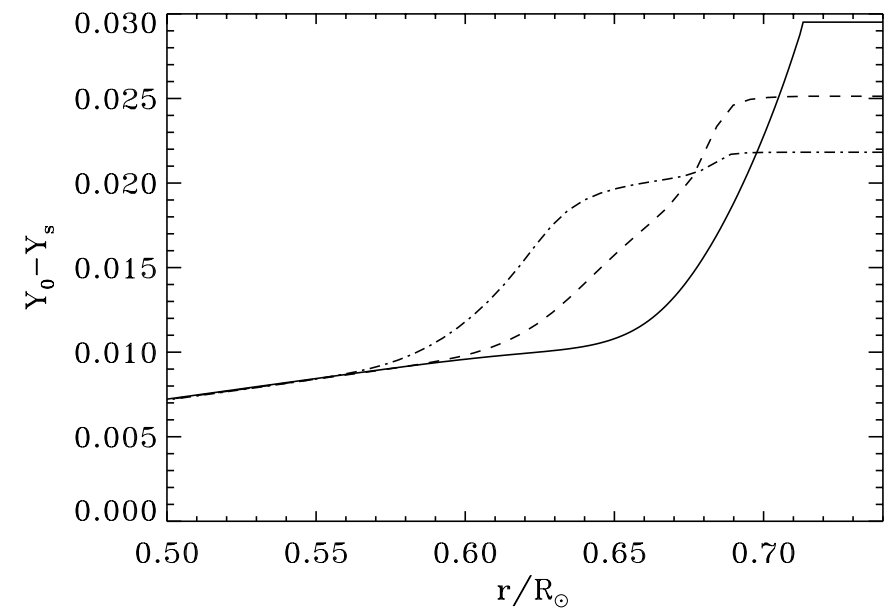

FIG. 2.- Radial profile of the difference of ${ }^{4} \mathrm{He}$ composition between the initial and present values for the reference solar model including only microscopic diffusion (solid line) and solar models where we add a macroscopic mixing owing to the presence of the tachocline: coefficient $A$ (dashed line) and $B$ (dash-dotted line; see $\S 3.3$ and Tables 1 and 3 for the model characteristics).

line). The value of $10 \mathrm{~cm}^{2} \mathrm{~s}^{-1}$ for $D_{i}$ is deduced from the expression of Michaud \& Proffitt (1993) for ${ }^{4} \mathrm{He}$ in the case of the reference solar model at the present age. It must be pointed out that in our calculation these microscopic diffusion coefficients as well as the microscopic velocity $v_{i}$ are evaluated for all the chemical species present in our gas mixture, and they are not assumed to be constant (see Paper I). The amplitude of $D_{4 \mathrm{He}}$ varies by only $10 \%$ in the range $[0.6,0.72] R_{\odot}$ and doesn't change significantly with time. The oscillating behavior of the macroscopic coefficient seen in Figure 1 reflects the subcell structure of the tachocline and reduces the lithium depletion, in comparison with a purely exponential coefficient, by a factor $\sim 1.5-2$ and induces stages in the radial chemical composition profile (see $\S 3.2$ and Fig. 2). The mixing in the tachocline thus depends on the following parameters for a given model of the present Sun:

1. the rotation rate $\Omega$, a measured quantity;

2. the differential rotation rate $\hat{\Omega}$ (or its projection $Q_{i}$ ) inferred from helioseismology;

3. the Brunt-Väisälä frequency $N$, determined by the extent of convective penetration; and

4. the horizontal diffusivity $v_{H}$, which presumably sides with the differential rotation rate.

There are at present no firm prescriptions available for $N$ and $v_{H}$, and therefore we must consider these, or two combinations of them, as free parameters in our calculations. We have chosen to keep $N$ and to take $d$ (twice the thickness of the tachocline), because both of them are constrained by upper limits drawn from helioseismology.

\section{SOLAR MODELS}

\subsection{Description of the Models}

In Paper I we calculated a new standard model using the CESAM code (Morel 1997) and discussed the validity of such a model in the light of the present helioseismic observations. We recall here only the main physical inputs present in this reference model. We used the solar chemical composition of Grevesse, Noels, \& Sauval (1996), the OPAL96 opacity tables of Iglesias \& Rogers (1996), the equation of state of Rogers, Swenson, \& Iglesias (1996), the recent updated nuclear reaction rates of Adelberger et al. (1998), the prescription of Mitler (1977) for the treatment of the nuclear screening reaction rates for a charge $Z>2$ (Dzitko et al., 1995), and the microscopic diffusion coefficients suggested by Michaud \& Proffitt (1993).

In the present paper (Paper II), we use the "pure microscopic diffusion" model as the reference model. We then compute solar models using the macroscopic coefficient $D_{T}$ described by equation (15) and follow the time evolution of these solar models from the pre-main sequence (PMS) until an age of $4.55 \mathrm{Gyr}$, making the age of our present Sun 4.6 Gyr (including about 0.05 Gyr of pre-main sequence). The adaptable time step increases from $\sim 20 \mathrm{yr}$ up to $200 \mathrm{Myr}$ during the evolution. We start from the PMS so as to properly follow the ${ }^{7} \mathrm{Li}$ abundance evolution, including the macroscopic diffusion coefficient along all the solar phases. We introduce an updated nuclear reaction rate for ${ }^{7} \mathrm{Li}(p, \alpha)^{4} \mathrm{He}$, using the Engstler et al. (1992) value instead of the Caughlan \& Fowler (1988) one. The results of the solar models we calculated for this study are summarized in Tables 1 and 2, and the photospheric abundances of the elements that are sensitive to the adding macroscopic process are compared with observations in Table 3.

TABLE 1

Solar Models With a Time-INDEPENDENT Macroscopic DIFFUSIVITY

\begin{tabular}{|c|c|c|c|}
\hline Parameters & Reference & $A$ & $B$ \\
\hline Opacities .............. & OPAL/A & OPAL/A & OPAL/A \\
\hline Diffusion .............. & yes & yes & yes \\
\hline Age $(\mathrm{Gyr}) \ldots \ldots \ldots \ldots \ldots$ & 4.6 & 4.6 & 4.6 \\
\hline$d\left(r / R_{\odot}\right) \ldots \ldots \ldots \ldots \ldots \ldots$ & $\cdots$ & 0.1 & 0.1 \\
\hline$N(\mu \mathrm{Hz}) \ldots \ldots \ldots \ldots \ldots$ & & 100 & 25 \\
\hline$(Z / X)_{s} \ldots \ldots \ldots \ldots \ldots \ldots$ & fixed & fixed & fixed \\
\hline$\alpha \ldots \ldots \ldots \ldots \ldots \ldots \ldots \ldots$ & 1.766 & 1.753 & 1.745 \\
\hline$Y_{0} \ldots \ldots \ldots \ldots \ldots \ldots \ldots$ & 0.2722 & 0.2703 & 0.2691 \\
\hline$Z_{0} \ldots \ldots \ldots \ldots \ldots \ldots \ldots$ & 0.01959 & 0.01919 & 0.01893 \\
\hline$(Z / X)_{0} \ldots \ldots \ldots \ldots \ldots \ldots$ & 0.0277 & 0.0270 & 0.0266 \\
\hline$Y_{s} \ldots \ldots \ldots \ldots \ldots \ldots \ldots \ldots \ldots$ & 0.2427 & 0.2452 & 0.2473 \\
\hline$Z_{s} \ldots \ldots \ldots \ldots \ldots \ldots \ldots$ & 0.01803 & 0.01797 & 0.01792 \\
\hline$(\dot{Z} / X)_{s} \ldots \ldots \ldots \ldots \ldots \ldots$ & 0.0245 & 0.0245 & 0.0245 \\
\hline$R_{b c z} / R_{\odot} \ldots \ldots \ldots \ldots \ldots$ & 0.7133 & 0.7145 & 0.7154 \\
\hline$T_{b c z} \times 10^{6}(\mathrm{~K}) \ldots \ldots \ldots$ & 2.190 & 2.181 & 2.174 \\
\hline$Y_{c} \ldots \ldots \ldots \ldots \ldots \ldots \ldots \ldots \ldots \ldots \ldots$ & 0.6405 & 0.6382 & 0.6367 \\
\hline$Z_{c} \ldots \ldots \ldots \ldots \ldots \ldots \ldots$ & 0.02094 & 0.02051 & 0.02024 \\
\hline$T_{c} \times 10^{6}(\mathrm{~K}) \ldots \ldots \ldots \ldots$ & 15.71 & 15.68 & 15.66 \\
\hline$\rho_{c}\left(\mathrm{~g} \mathrm{~cm}^{-3}\right) \ldots \ldots \ldots \ldots$ & 153.1 & 152.9 & 152.7 \\
\hline${ }^{71} \mathrm{Ga}(\mathrm{SNU}) \ldots \ldots \ldots \ldots$ & 127.1 & 126.0 & 125.2 \\
\hline${ }^{37} \mathrm{Cl}(\mathrm{SNU}) \ldots \ldots \ldots \ldots . .$. & 7.04 & 6.84 & 6.71 \\
\hline${ }^{8} \mathrm{~B}\left(10^{6} \mathrm{~cm}^{-2} \mathrm{~s}^{-1}\right) \ldots \ldots$ & 4.99 & 4.83 & 4.73 \\
\hline
\end{tabular}

NoTE.-The models include a tachocline mixing with different values of the Brunt-Väisälä frequency and of the tachocline thickness and arc compared to the reference model. Parameter definitions: $d$ is twice the tachocline thickness $h ; N$ is the Brunt-Väisälä frequency; $\alpha$ is the mixing-length parameter, $Y_{0}, Z_{0},(Z / X)_{0}$ are the initial helium, heavy element, and ratio heavy element on hydrogen; $Y_{s}, Z_{s}$, and $(Z / X)_{s}$ are the same for surface compositions; $R_{\mathrm{bzc}}$ and $T_{\mathrm{bcz}}$ are the radius and temperature at the base of the convective zone; $Y_{c}, Z_{c}, T_{c}, \rho_{c}$ are the central helium, heavy-element contents, central temperature and density; and ${ }^{71} \mathrm{Ga},{ }^{37} \mathrm{Cl},{ }^{8} \mathrm{~B}$ are the respective neutrino predictions for the gallium, the chlorine, and the water detectors. OPAL/A means that we use OPAL96 opacities and Alexander \& Ferguson 1994 for low temperature. 
TABLE 2

Solar Models with a Time-DEPENDENT Macroscopic Diffusivity

\begin{tabular}{|c|c|c|c|c|c|}
\hline Parameters & Reference & $A_{t}$ & $B_{t}$ & $B_{t z}$ & $C_{t}$ \\
\hline Opacities .............. & OPAL/A & OPAL & OPAL/A & OPAL/A & OPAL/A \\
\hline Diffusion .............. & yes & yes & yes & yes & yes \\
\hline Age $(\mathrm{Gyr}) \ldots \ldots \ldots \ldots \ldots$ & 4.6 & 4.6 & 4.6 & 4.6 & 4.6 \\
\hline$d\left(r / R_{\odot}\right) \ldots \ldots \ldots \ldots \ldots \ldots$ & $\ldots$ & 0.1 & 0.1 & 0.1 & 0.05 \\
\hline$N(\mu \mathrm{Hz}) \ldots \ldots \ldots \ldots \ldots \ldots$ & $\ldots$ & 100 & 25 & 25 & 25 \\
\hline$(Z / X)_{s} \ldots \ldots \ldots \ldots \ldots \ldots \ldots$ & fixed & fixed & fixed & free & fixed \\
\hline 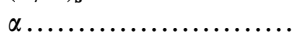 & 1.766 & 1.752 & 1.743 & 1.755 & 1.748 \\
\hline$Y_{0} \ldots \ldots \ldots \ldots \ldots \ldots \ldots$ & 0.2722 & 0.2701 & 0.2689 & 0.2722 & 0.2695 \\
\hline $\mathrm{i}_{0} \ldots \ldots \ldots \ldots \ldots \ldots \ldots \ldots$ & 0.01959 & 0.01914 & 0.01889 & 0.01959 & 0.01903 \\
\hline$(Z / X)_{0} \ldots \ldots \ldots \ldots \ldots \ldots$ & 0.0277 & 0.0269 & 0.0265 & 0.0277 & 0.0267 \\
\hline$Y_{s} \ldots \ldots \ldots \ldots \ldots \ldots \ldots \ldots$ & 0.2427 & 0.2455 & 0.2477 & 0.2510 & 0.2464 \\
\hline$Z_{s} \ldots \ldots \ldots \ldots \ldots \ldots \ldots$ & 0.01803 & 0.01796 & 0.01791 & 0.01858 & 0.01794 \\
\hline$(Z / X)_{s} \ldots \ldots \ldots \ldots \ldots \ldots$ & 0.0245 & 0.0245 & 0.0245 & 0.0255 & 0.0245 \\
\hline$R_{b c z} / R_{\odot} \ldots \ldots \ldots \ldots \ldots \ldots$ & 0.7133 & 0.7146 & 0.7155 & 0.7141 & 0.715 \\
\hline$T_{b c z} \times 10^{6}(\mathrm{~K}) \ldots \ldots \ldots \ldots$ & 2.190 & 2.180 & 2.173 & 2.194 & 2.177 \\
\hline$Y_{c} \ldots \ldots \ldots \ldots \ldots \ldots \ldots \ldots$ & 0.6405 & 0.6379 & 0.6365 & 0.6405 & 0.6372 \\
\hline$Z_{c} \ldots \ldots \ldots \ldots \ldots \ldots \ldots \ldots$ & 0.02094 & 0.02047 & 0.02019 & 0.02094 & 0.02034 \\
\hline$T_{c} \times 10^{6}(\mathrm{~K}) \ldots \ldots \ldots \ldots$ & 15.71 & 15.67 & 15.65 & 15.71 & 15.66 \\
\hline$\rho_{c}\left(\mathrm{~g} \mathrm{~cm}^{-3}\right) \ldots \ldots \ldots \ldots$ & 153.1 & 152.8 & 152.7 & 153.1 & 152.8 \\
\hline${ }^{71} \mathrm{Ga}(\mathrm{SNU}) \ldots \ldots \ldots \ldots$. & 127.1 & 125.9 & 125.1 & 127.1 & 125.5 \\
\hline${ }^{37} \mathrm{Cl}(\mathrm{SNU}) \ldots \ldots \ldots \ldots \ldots$ & 7.04 & 6.82 & 6.69 & 7.04 & 6.76 \\
\hline${ }^{8} \mathrm{~B}\left(10^{6} \mathrm{~cm}^{-2} \mathrm{~s}^{-1}\right) \ldots \ldots$ & 4.99 & 4.81 & 4.70 & 4.99 & 4.76 \\
\hline
\end{tabular}

NoTE.-The models include a tachocline mixing with different values of the Brunt-Väisälä frequency and of the tachocline thickness and are compared to the reference model. Model $B_{t z}$ shows the effect of noncalibrated $Z / X$ (see text and Table 1 for the parameter definitions).

\subsection{Time-independent Rotation}

We first compute solar models that include the effective diffusivity $D_{T}$ (eq. [15]) with a tachocline thickness $h$ of 0.05 $R_{\odot}\left(d=0.1 R_{\odot}\right)$, in agreement with the helioseismic measurements (Basu 1997; Corbard et al. 1998, 1999; Charbonneau et al. 1998); a Brunt-Väisälä frequency $N$ of 100 or 25 $\mu \mathrm{Hz}$, to take into account the rapid variation of this quantity at the base of the convective zone; and a rotation $\Omega$ at the base of the convective zone of $0.415 \mu \mathrm{Hz}$; we call these coefficients $A$ and $B$, respectively (see Table 1 ). The reference model has a present photospheric helium abundance of 0.2427 in mass, corresponding to an ${ }^{4} \mathrm{He}$ diffusion of $10.8 \%$. This value of $Y_{s}$ is slightly too low if we compare with the Basu \& Antia (1995) value for the OPAL equation of state, $Y_{s}=0.249 \pm 0.003$.

When introducing the macroscopic diffusion coefficient, we mix back helium in the convection zone, inhibiting the microscopic diffusion by $15 \%$ or $25 \%$ and leading to a present photospheric ${ }^{4} \mathrm{He}$ abundance $Y_{s}=0.2452$ or $Y_{s}=$ 0.2473 , respectively, for models $A$ and $B$ (see Tables 1 and 3; Brun, Turck-Chièze \& Zahn 1998). This is in better agreement with the helioseismic values. The decrease of the Brunt-Väisälä frequency $N$ leads to an increase of the efficiency of the mixing in the tachocline layer and reduces the settling of the chemical species. We also note that the initial helium content $Y_{0}$ is smaller than 0.27 in mass (see Table 1 and also Paper I).

In Figure 2 we present the difference between the initial helium composition, $Y_{0}$, and the present photospheric one, $Y_{s}$, for several models, including the reference one. Before the introduction of microscopic diffusion, this difference was null because the present photospheric values were adopted as the initial composition of the star. However, now when we compute a standard model including the microscopic diffusion and the settling of chemical species, the initial abundances are assumed to be different from their

TABLE 3

PHOTOSPHERIC ABUNDANCES

\begin{tabular}{|c|c|c|c|c|c|c|c|c|c|}
\hline Parameter & Observaton & Reference & $A$ & $B$ & $A_{t}$ & $B_{t}$ & $B_{t z}$ & $B_{t o}$ & $C_{t}$ \\
\hline$\ldots \ldots \ldots$ & $\ldots$ & $\ldots$ & 100 & 25 & 100 & 25 & 25 & 25 & 25 \\
\hline${ }^{4} \mathrm{He}_{s} \ldots \ldots \ldots \ldots \ldots$ & $0.249 \pm 0.003$ & 0.2427 & 0.2452 & 0.2473 & 0.2455 & 0.2477 & 0.2509 & 0.2477 & 0.2464 \\
\hline$(\mathrm{Z} / \mathrm{X})_{s} \ldots \ldots \ldots \ldots \ldots$ & $0.0245 \pm 0.002$ & 0.0245 & 0.0245 & 0.0245 & 0.0245 & 0.0245 & 0.0255 & 0.0245 & 0.0245 \\
\hline${ }^{7} \mathrm{Li}_{0} /{ }^{7} \mathrm{Li}_{s} \ldots \ldots \ldots \ldots$ & $\sim 100$ & $\sim 6$ & $\sim 8$ & $\sim 22$ & $\sim 12$ & $\sim 91$ & $\sim 134$ & $\sim 64$ & $\sim 89$ \\
\hline
\end{tabular}

NotE.-The table shows the surface abundance variation of ${ }^{3} \mathrm{He} /{ }^{4} \mathrm{He}$ during the last $3 \mathrm{Gyr}$, surface abundances of ${ }^{4} \mathrm{He}$ and heavy elements $Z$, and the initial/surface abundance ratio for ${ }^{7} \mathrm{Li}$ and ${ }^{9} \mathrm{Be}$ from observations and for solar models at the solar age (subscript $t$ is for time-dependent models, $t z$ is for the time-dependent model with $Z_{0}=Z_{0}^{\text {ref }}=0.01959$, and to is for the time-dependent model with the Caughlan \& Fowler $19888^{7} \mathrm{Li}(p, \alpha)^{4} \mathrm{He}$ nuclear reaction rate). 
present day values, and there is an iteration on the composition to get the photospheric $Z / X$ ratio in agreement with the one measured at the present time: $Z / X=0.0245 \pm 0.003$.

The prominent feature of Figure 2 is the smoother composition profile for models including the macroscopic diffusivity $D_{T}$, in comparison with the reference model, which presents a sharp composition gradient just below the convective zone. The composition profile is also almost constant below the convective zone over a distance that is related with the value of $d$ and $N$. A smaller Brunt-Väissälä frequency value induces a greater mixing and then an extended plateau. The characteristic steps in the radial profile come from the cosine term in the expression of $D_{T}$ (eq. [15]; see also Elliott 1997). An explanation could be that the simplified assumption in the treatment of the tachocline of SZ92, of a constant turbulent horizontal viscosity $v_{H}$, induces this radial dependence of the coefficient $D_{T}$ and thus the steps in the composition profile. A more careful study should be done to verify this statement.

Concerning the ${ }^{7} \mathrm{Li}$ and ${ }^{9} \mathrm{Be}$ depletion, we see in Table 3 that the mixing increases the lithium depletion by a factor up to $\sim 4$, without burning ${ }^{9} \mathrm{Be}$, which agrees with the revised determination by Balachandran \& Bell (1998). Nevertheless, such an increase of the ${ }^{7} \mathrm{Li}$ depletion is still insufficient to reproduce the photospheric lithium depletion (Grevesse et al. 1996; Cayrel 1998 and references therein).

\subsection{Time-dependent Rotation}

In this section we shall take into account the time evolution of the tachocline width and the efficiency of the mixing from the early phase of the Sun until now. In the previous section we used a constant value for $d$ based on the present observations of the tachocline thickness in the effective diffusion coefficient $D_{T}$. Thus, in order to improve the description of tachocline mixing, we now introduce a time dependence of the coefficient $D_{T}$, and of the tachocline thickness $h$ (and hence $d$ ), related to the global and the differential rotation of the Sun as follows:

$$
D_{T} \propto v_{H}\left(\frac{d}{r_{\mathrm{bcz}}}\right)^{2} Q_{i}^{2} \propto \Omega v_{H}^{1 / 2}\left(\frac{\hat{\Omega}}{\Omega}\right)^{2}, \quad d \propto \frac{\Omega^{1 / 2}}{v_{H}^{1 / 4}},
$$

where we have used equations (15) and (11). Assuming that the turbulent viscosity is proportional to the differential rotation (i.e., $v_{H} \propto \hat{\Omega}$ ) and introducing the dependence of differential rotation on rotation observed by Donahue, Saar, \& Baliunas (1996) $\left(\hat{\Omega} \propto \Omega^{0.7 \pm 0.1}\right)$, we finally obtain for $D_{T}(\Omega)$ and $d(\Omega)$ the following scaling laws:

$$
D_{T} \propto \Omega^{0.75 \pm 0.25}, \quad d \propto \Omega^{(1.3 \mp 0.1) / 4} .
$$

We conclude that the tachocline mixing was stronger in the past both because that layer was thicker and because the diffusivity was larger. Note that the observational uncertainties in the relation between $\hat{\Omega}$ and $\Omega$ act in opposite way on the dependence of $D_{T}(\Omega)$ and $d(\Omega)$ (i.e., a stronger mixing implies a smaller tachocline extent). We render the mixing in the tachocline time-dependent, through $D_{T}[\Omega(t)]$ and $d[\Omega(t)]$, by using the spin-down law $\Omega(t) \propto t^{-1 / 2}$ that was deduced by Skumanich (1972) from the rotation rate of stellar clusters of different ages.

Models $A_{t}, B_{t}$, and $C_{t}$ correspond to solar evolutions with time-dependent coefficients given by equation (17) and different values for $N$ and the present tachocline thickness $d$ (see Table 1 and 2). The first two cases correspond to the same values of $d$ and $N$ that existed in the previous section, but with a time dependence of the coefficient $D_{T}$. The third shows the influence of a thinner tachocline $\left(d=0.05 R_{\odot}\right)$, as suggested by more recent studies (Corbard et al. 1999). The introduction of this time dependence increases the inhibition of the microscopic diffusion of helium during the evolution in comparison with the previous solar models considered in section 3.2 (see Table 3). These models show an inhibition of ${ }^{4} \mathrm{He}$ up to $27 \%$ for model $B_{t}(d=0.1$, $N=25$ ) leading to a surface helium $Y_{s}=0.2477$, very close to the seismic value of Basu \& Antia (1995).

We plot the ${ }^{7} \mathrm{Li}$ and ${ }^{9} \mathrm{Be}$ radial profiles in Figure 3, for the reference model and the time-dependent models calibrated in $Z / X$ at $4.6 \mathrm{Gyr}$, with the corresponding radial temperature scale. These two light chemical elements are sensitive to mixing processes occurring in the stars because their nuclear burning temperatures are very low (respectively, 2.5 and $3.2 \times 10^{6} \mathrm{~K}$; Baglin \& Lebreton 1990). It is well known that the solar ${ }^{7} \mathrm{Li}$ is depleted by a factor $\sim 100$ (Grevesse et al. 1996), and recent revised abundance determinations have shown that ${ }^{9} \mathrm{Be}$ abundance is practically undepleted (Balachandran \& Bell 1998) contrary to what was thought previously. These new observational constraints can only be satisfied if those chemical species are mixed in a rather thin layer below the convective zone, which is the case for the tachocline mixing considered here.

We clearly see in Figure 3 that this mixing process modifies the distribution of lithium but not of beryllium (except the flat plateau for the mixed models in comparison with the "pure" diffusion one). These results are confirmed in Table 3, where we show the initial over present ratio of the ${ }^{7} \mathrm{Li}$ and ${ }^{9} \mathrm{Be}$. We also remark that models including a macroscopic diffusivity with a low value of the BruntVäisälä frequency $N=25$ (i.e., $B_{t}$ and $C_{t}$ ) burn more ${ }^{7} \mathrm{Li}$, because of their higher absolute value $\left(\geq 10^{5} \mathrm{~cm}^{2} \mathrm{~s}^{-1}\right)$ in comparison with the microscopic diffusion one $\left(\sim 10 \mathrm{~cm}^{2}\right.$ $\mathrm{s}^{-1}$ ), than models with a higher $N=100$ (i.e., $A_{t}$ ) (Fig. 1).

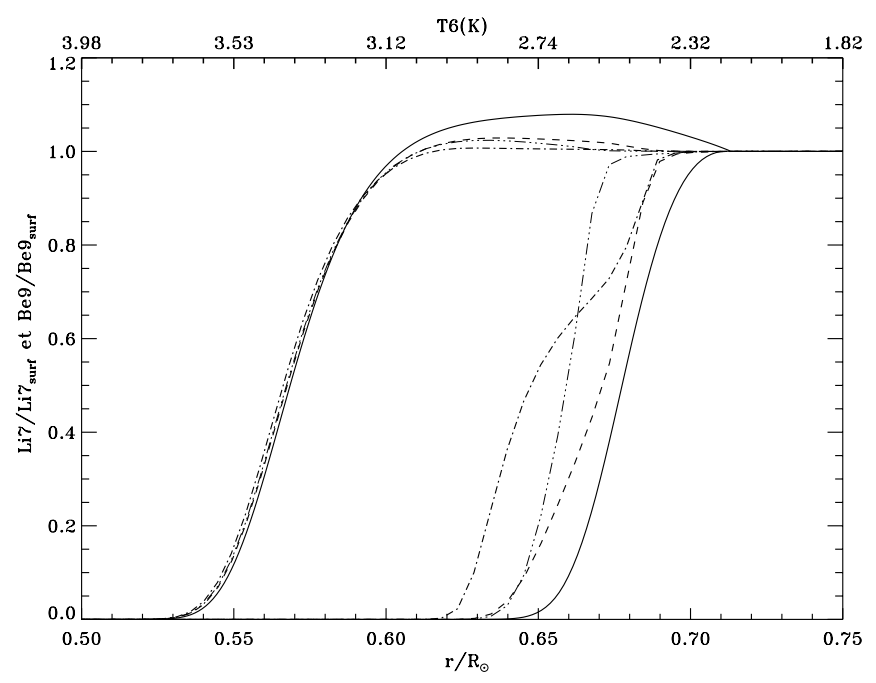

FIG. 3.- ${ }^{7} \mathrm{Li}$ and ${ }^{9} \mathrm{Be}$ radial profile for several models (superimposed on the corresponding temperature $T_{6}=T / 10^{6} \mathrm{~K}$ obtained for the reference model). Models: reference (solid line), $A_{t}$ (dashed line), $B_{t}$ (dash-dotted line), and $C_{t}$ (dash-triple-dotted line). The mixing below the convective zone modifies only the ${ }^{7} \mathrm{Li}$ profile and not the ${ }^{9} \mathrm{Be}$ one (except the flat plateau for the mixed models). 


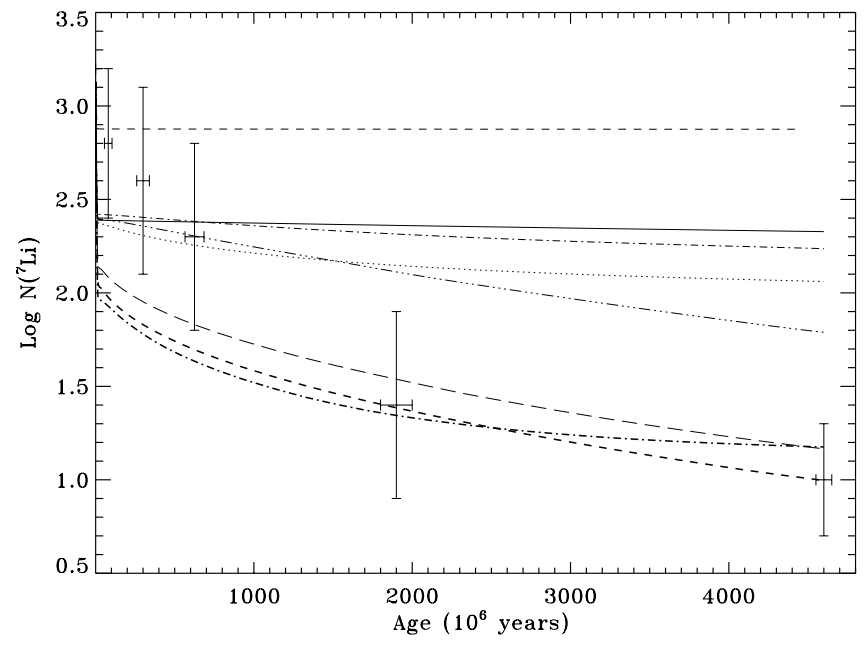

Fig. 4.-Time-dependent depletion of ${ }^{7} \mathrm{Li}$ for several solar models. Models with no microscopic diffusion (dashed line), with microscopic diffusion (solid line), and with mixing in the tachocline thickness: $A$ (dash-dotted line); $B$ (dash-triple-dotted line). Models with time-dependent mixing: $A_{t}$ (dotted line), $B_{t}$ (long-dashed line), $C_{t}$ (thick dash-dotted line), and $B_{t z}\left(Z_{0}=\right.$ $\left.Z_{0}^{\text {std }}=0.01959\right)$ (thick dashed line). We superimpose as error-barred points on the theoretical curves the following open cluster observations: Pleiades (Soderblom et al. 1993b); UMaG (Soderblom et al. 1993a); Hyades (Thorburn et al. 1993); NGC 752 (Balachandran 1995); and the solar one at the present age. (Figure adapted from Vauclair \& Richard 1998 and cluster age uncertainties deduced from Lebreton et al. 1997 and private communication [1999].)

The models $B_{t}$ and $C_{t}$ strongly inhibit the settling of the chemical species and mix all the elements over a distance where the lithium burning temperatures are reached $r \sim$ $0.67 R_{\odot}$. The characteristic time for this mixing for a $D_{T}$ of $10^{5} \mathrm{~cm}^{2} \mathrm{~s}^{-1}$ is $\sim 3 \mathrm{Myr}$. This time is intermediate between the convective cell turnover time $(\sim 1$ month) and the microscopic diffusion time ( $>10 \mathrm{Gyr})$. We also see in Table 3 that the time-dependent diffusivity improves the ${ }^{7} \mathrm{Li}$ depletion, where a value of $\sim 100$ is obtained without destroying ${ }^{9} \mathrm{Be}$ and without increasing the ${ }^{3} \mathrm{He} /{ }^{4} \mathrm{He}$ surface ratio over the past $3 \mathrm{Gyr}$, as deduced by Geiss \& Gloeckler (1998) from meteorites and solar wind abundance measurements. As already mentioned, these abundance constraints can be satisfied only if the mixing occurs in a thin layer below the convective zone, which is the case for the tachocline considered here.

In Figure 4 we present the lithium depletion occurring during the Sun evolution for the different models presented, plus one without microscopic diffusion. Clearly, only the diffusion models including mixing in the tachocline show a substantial depletion during the main-sequence evolution as it is observed in different clusters. One notes that the lithium depletion is largely dependent on the BruntVäissälä frequency adopted at the base of the convective zone and on the time dependence of the macroscopic coefficient. The modification of the tachocline thickness has only a little impact on the lithium depletion, as the higher value of the coefficient largely compensates the reduction of the thickness. However, one sees that the ${ }^{7} \mathrm{Li}$ depletion slopes in these two models (i.e., $B_{t}$ and $C_{t}$ ) are not the same. The strong time-dependent mixing with $N=25$ presents a correct value of the solar ${ }^{7} \mathrm{Li}$ depletion. The lithium depletion during the PMS is probably overestimated owing to the crude spin-down law we have adopted. A more detailed analysis of these phases is under study (see also Vauclair \& Richard 1998; Montalban \& Schatzman 1996).

\section{DISCUSSION}

\subsection{The Sound-Speed Profile}

We now compare the sound-speed profile of the different models with the helioseismic inversion results obtained with the GOLF + MDI data (Lazrek et al. 1997; Roca Cortes et al. 1998; Kosovishev et al. 1997) and described in TurckChièze et al. (1997, 1998). We see in Figure 5 that the peak localized at $r \sim 0.68 R_{\odot}$ is practically erased by this process, depending on the strength of the mixing.

In all the models shown in Figure 5, we have adjusted the initial composition in order to get at the solar age, the present observed photospheric heavy-element/hydrogen ratio, i.e., $Z / X=0.0245$. This calibration causes the swell of the small bump located between the nuclear core and the convective zone, since it reduces the initial metallicity (see Table 2). It is well known that the heavy-element abundance has a direct influence on the solar structure, mainly through the opacity coefficients, and hence it influences the sound-speed profile between 0.4 and $0.7 R_{\odot}$ (Turck-Chièze 1995, 1998; Paper I). The result is quite different if we do not constrain the photospheric $Z / X$ value of the mixed model. Starting with the heavy-element composition $Z_{0}=$ 0.01959 of the reference model, for example, we obtain a different chemical composition at the solar age (see, for example, the third and fourth columns of Table 2 for the coefficients $B_{t}$ and $B_{t z}$ ), but this composition is still compatible with the observational data. In Figure 6 we display the corresponding squared radial sound-speed differences between the models and the induced solar value for two models where we have kept (i.e., coefficient $B_{t}$ ) or relaxed $\left(B_{t z}\right)$ this surface heavy-element constraint. We see that in both cases where the macroscopic term is introduced, the bump around $0.68 R_{\odot}$ is practically erased by the introduction of the tachocline mixing, but the calibrated model is modified along the whole structure and not only close to the bump, which is not the case for the noncalibrated model where $Z_{0}$ is fixed. This is illustrated also by Figure $7 a$, where the noncalibrated model changes only locally the structure of the solar model (dash-dotted line).

A similar result is obtained by Elliott, Gough, \& Sekii (1998) by invoking an advection of the heavy elements at

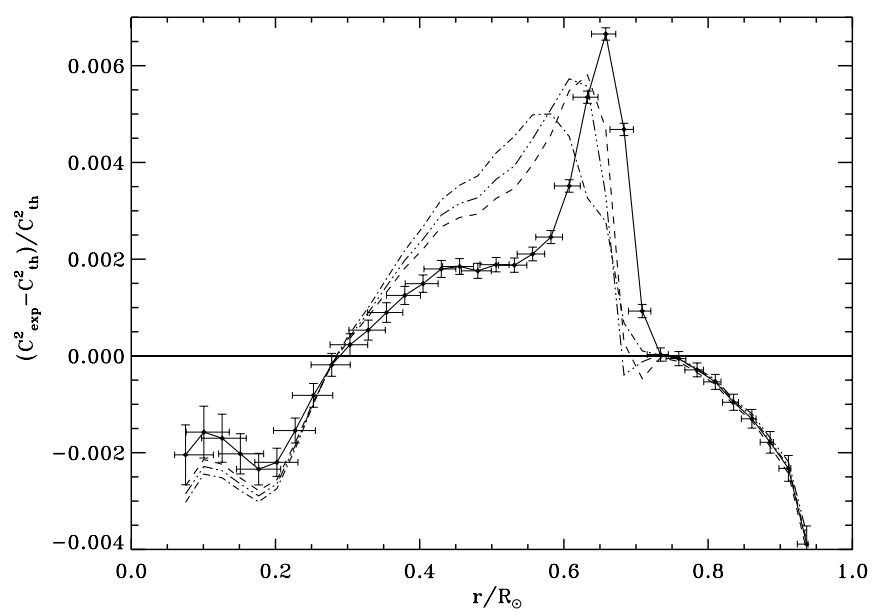

FIG. 5.- Sound-speed square difference between GOLF + MDI data, the reference model (solid line) and models including a macroscopic term: $A_{t}$ (dashed line), $B_{t}$ (dash-dotted line), $C_{t}$ (dash-triple-dotted line) (see parameters in Table 2). 


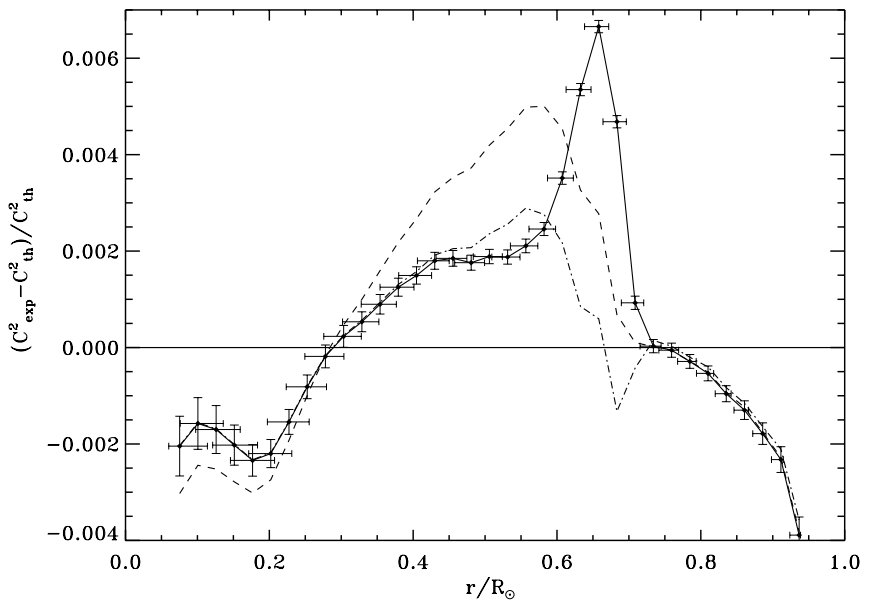

FIG. 6.- Sound-speed square difference between GOLF + MDI data, the reference model (solid line) and two models including a macroscopic term: $B_{t}$ calibrated in $Z / X$ (dashed line) and $B_{t z}$ with a noncalibrated $Z_{0}=Z_{0}^{\text {std }}=0.01959($ dash-dotted line $)$.

the base of the convection zone. They deduced from the sound-speed profile a low value of the tachocline thickness $(h=0.02)$, which could be due to the macroscopic coefficient used. In this estimate, they did not calibrate their model in $Z / X$ and obtained a result similar to ours (Fig. 7a, dash-dotted line).

\subsection{Solar Composition}

Examining the different observables, it seems that the model using the coefficient $B_{t z}$ (see Tables 2 and 3) corresponds today to a good representation of the present Sun. The agreement on the sound speed is significantly improved and the observed photospheric light elements $\left({ }^{3} \mathrm{He},{ }^{4} \mathrm{He}\right.$, ${ }^{7} \mathrm{Li}$, and ${ }^{9} \mathrm{Be}$ ) are well reproduced. In this model, two effects contribute to this improvement: the introduction of the mixing at the base of the convection zone, which partly inhibits the microscopic diffusion; and the induced increase of the heavy elements at the surface and consequently in the intermediate region due to this inhibition. This model treats the tachocline instability as a local phenomenon.

In Figure $7 b$ we show the heavy-element profile along the solar radius of the reference model and the mixed models

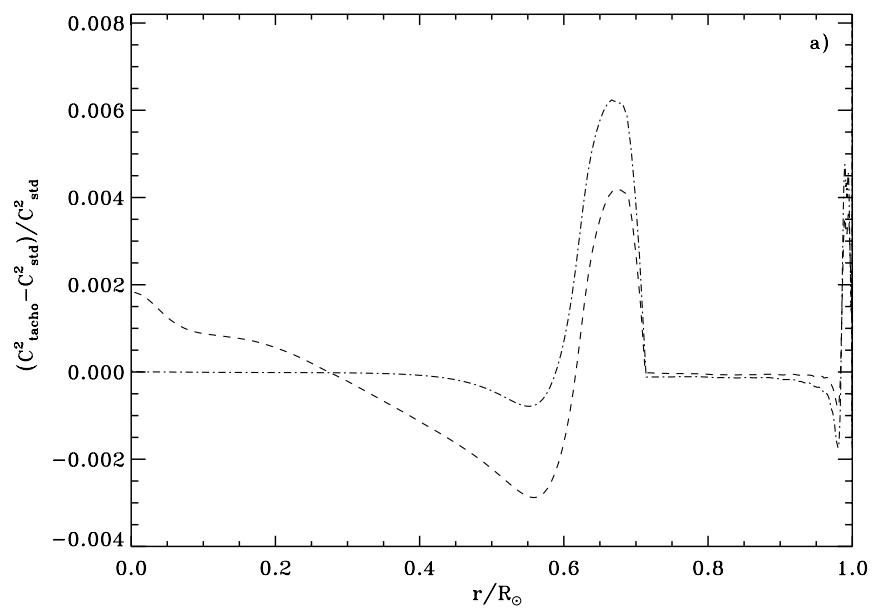

(coefficients $B_{t} \& B_{t z}$ ) with and without the $Z / X=0.0245$ calibration. We clearly see that without the $Z / X$ calibration there is a difference in the $Z$ profile, corresponding to an increase of the opacity and then to a reduction of the disagreement between the seismic sound speed and the theoretical one.

In Paper I we had already mentioned that the soundspeed difference between the Sun and the model may suggest that a slight increase of the heavy-element composition of about $5 \%$ is required. One notes that the results we obtain without specific calibration, but keeping the initial heavy-element composition of the reference model, lead to a photospheric heavy-element composition of $Z / X=0.0255$. This is still consistent with the present observational uncertainties (about $10 \%$ ), even if a very recent work seems to suggest a possible reduction of the $\mathrm{CNO}$ abundances rather than an slight increase (Grevesse \& Sauval 1998). We have verified this statement by performing a "pure" microscopic model with $Z / X=0.0255$. If one compares the sound-speed profile of model $B_{t z}$ (dot-dashed line) to the reference model with $Z / X=0.0245$ (Fig. 6), it shows a better overall agreement with the seismic data. Otherwise, if one compares this model to a "pure" microscopic model with $Z / X=0.0255$, one reaches a different conclusion for the radiative part (identical to the comparison of model $B_{t}$ with the reference model Fig. 6). This intricate process shows that it is not so easy to find the real behavior of the Sun. If we increase the initial heavy-element abundance a little more, we destroy the agreement of the tachocline region and obtain a photospheric composition that is not in as good agreement with the present observations. So, extensive studies are surely useful to properly disentangle the competing processes.

The remaining part of the sound-speed discrepancy located between the nuclear core and the convection zone (see Fig. 6) may come from uncertainties in the equation of state or opacity coefficients (Turck-Chièze et al. 1998) or from our simplified treatment of the microscopic diffusion. In the present study, we do not consider the different states of ionization of the different species or the radiative acceleration in the calculation of the microscopic diffusivity (Turcotte et al. 1998). We also cannot exclude the presence of other mixing processes, for example, the meridional circulation or the effect of the internal waves or magnetic field.

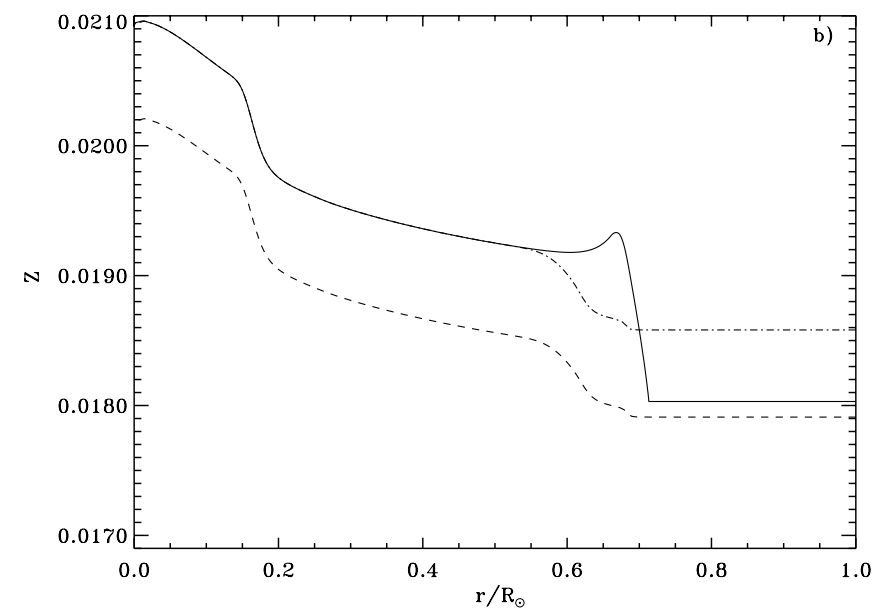

FIG. 7.- (a) The effect of calibration on the sound-speed square difference between the reference model and two models including a macroscopic term: $B_{t}$ calibrated in $Z / X$ (dashed line) and $B_{t z}$ with a noncalibrated $Z_{0}=Z_{0}^{\text {std }}=0.01959$ (dash-dotted line). (b) Heavy-element composition for the reference model including only microscopic diffusion (solid line) and two models with macroscopic mixing due to the tachocline: $B_{t}$ calibrated in $Z / X$ (dashed line) and $B_{t z}$ with a noncalibrated $Z_{0}=Z_{0}^{\text {std }}=0.01959$ (dash-dotted line). (See Table 2 for the model characteristics.) 
It seems difficult to think that the residual discrepancy comes from insufficient knowledge of the element abundances, as an increase of the heavy-element composition will have a dramatic effect in the region of the energy transport transition.

\subsection{Nuclear Reaction Rates}

The depletion of lithium is of course dependent on the nuclear reaction rates used. In this study, we have used the recent compilation of the NACRE consortium (Angulo et al. 1999), which recommends the nuclear reaction rate ${ }^{7} \mathrm{Li}(p, \alpha)^{4} \mathrm{He}$ of Engstler et al. (1992). The astrophysical factor is increased by $30 \%$ in comparison with the older value recommended by Caughlan \& Fowler (1988), mainly owing to a higher accuracy numerical integration. The updated value of $S_{71}(0)=0.0593 \mathrm{MeV}$ barn increases the lithium depletion during the Sun evolution by $\sim 40 \%$ (see Table 3 models $B_{t o}$ and $B_{t}$ ). This effect is introduced in all the results presented in this study and constitutes an important point that must be considered in the lithium depletion problem in stars. It is interesting also to notice that the reaction rate of the ${ }^{7} \mathrm{Li}(p, \gamma)^{8} \mathrm{~B}$, which is not included in our study, mainly because this cross section was considered as very small, has been reestimated these last years and has been found to be 100 times greater than in Caughlan \& Fowler (1988). The impact on the present study must be smaller than $10 \%$, considering the relative importance of the two reactions.

\subsection{Neutrino Predictions}

Of course, one issue of improving solar models is to predict more correctly the neutrino fluxes produced in the central region of the Sun (Turck-Chièze 1999). Tables 1 and 2 show the predicted values for all the models considered. One notices a very small dependence on the macroscopic coefficient used but, nevertheless, two kinds of results, depending on whether the models are calibrated in $Z / X$ or not. This difference is connected to the effect of the inhibition of the microscopic processes, which leads to a slight decrease of the neutrino fluxes.Indeed, we observe, in the calibrated models, a reduction in comparison with the reference model of $-1.6 \%$ for the GALLEX (Gallium Experiment) and SAGE (Soviet-American Gallium Experiment) experiments (respectively, Hampel et al. 1996 and Abdurashitov et al. 1996), of $-5.1 \%$ for the Homestake experiment (Cleveland et al. 1998) and $-5.6 \%$ for SuperKamiokande (Suzuki et al. 1998). It is useful to recall that the general effect of the introduction of the microscopic diffusion in solar model was an increase of about $20 \%$ on the ${ }^{8} \mathrm{~B}$ neutrino flux prediction, so this corresponds to $25 \%$ of this effect. But one may also notice that the effect of inhibition could be exactly compensated by the effect of the increase of the heavy elements by $5 \%$ (reference model and model $B_{t z}$ ).

These effects are too small to solve the neutrino problem. This is hardly a surprise, since the tachocline mixing occurs at the base of the convection zone and hence is too remote to have a serious impact on the structure of the nuclear core. Even if this study is not crucial for the neutrino fluxes, it gives certainly a proper description of the chemical transport of elements in the external part of the Sun and definitively excludes a large increase of the neutrino fluxes by this kind of processes as was thought five years ago. The introduction of a macroscopic diffusivity also destroys the criti- cisms on the impact of the pure microscopic diffusion, which was well known to be partly inhibited by some turbulent processes and which seems now well under control, at least as far as the extension in the Sun is concerned. We begin to include dynamical effects properly in stellar models, and one cannot exclude that the cumulative effect of different modifications of the physical inputs (microscopic and macroscopic) may lead to smaller neutrino fluxes. We will not comment, in this paper, on the discrepancy of the sound speed in the central region because this is more difficult to estimate (Turck-Chièze \& Basu 1998; Gonczi et al. 1998) and to interpret there, owing to the sensitivity of the sound speed to a lot of competing processes (see Paper I). Works are in progress to have good constraints on the core.

\section{CONCLUSION}

In this paper, we have shown that the presence of mixing in the tachocline layer partly inhibits the microscopic diffusion process and improves the agreement with the helioseismic and photospheric abundance data. Our results make use of updated helioseismic constraints on the present extent of this layer, and they are based on a physical treatment of the tachocline layer (SZ92) that involves only one additional parameter, namely the Brunt-Väisälä frequency (which we assume constant in our model). The solar models thus obtained are quantitatively in good agreement with the observational data, and our favored model is model $B_{t z}$ (see Tables $2 \& 3$ ). When introducing a time-dependent tachocline mixing, it is possible to reach the observed ${ }^{7} \mathrm{Li}$ photospheric abundance at the present solar age without destroying ${ }^{9} \mathrm{Be}$ or bringing too much ${ }^{3} \mathrm{He}$ to the surface. This does not necessarily mean that our mixing process is the only one that operates in the Sun: others may contribute, such as mixing by gravity waves (Montalban \& Schatzman 1996) or meridional circulation (Zahn 1992; Vauclair \& Richard 1998). But the lack of depletion of ${ }^{9}$ Be proves that the mixing occurs in a rather thin layer below the convective zone, which is the case in our models. The model that seems most promising assumes a Brunt-Väisälä frequency $N=25 \mu \mathrm{Hz}$; it leads to a slight increase of the heavy-element abundance by $5 \%$ at the surface, compatible with the present uncertainties. A detailed study of the feedback of such mixing on the structure of the Sun reveals that its effect is not confined to the base of the convection zone but that it spreads into the region below, except if one relaxes the fixed $Z / X$ constraint and let the model adjusts within the heavy-element abundance incertitudes (model $B_{t z}$.

From the results of Papers I and II, we could deduce a Galactic enrichment of ${ }^{4} \mathrm{He}$. We find that the solar ${ }^{4} \mathrm{He}$ primordial abundance is $Y_{0}=0.27_{-0.001}^{+0.002}$ and leads to an enrichment of $10.6_{-1.3}^{+2.8} \%$ from the value of $0.244 \pm 0.002$ deduced by Thuan \& Izotov (1998) from observations of a large sample of low-metallicity $\mathrm{H}$ in regions in blue compact dwarf galaxies.

This first attempt to calibrate dynamical processes with helioseismology data from $\mathrm{SOHO}$ leads to the idea that the neutrino problem could be seen from a new angle by considering reliable solar models with macroscopic processes, which have not been implemented previously. The process invoked here concerns the $5 \%$ part of the external mass and consequently has little impact on neutrino predictions. But even if the solar models are close to the seismic data for 
$r<0.2 R_{\odot}$, the remaining discrepancies are still significant $(\sim$ few $\sigma)$ in comparison with seismic uncertainties, and they suggest that other physical processes, neglected so far, may operate there.

To conclude, this study encourages the introduction of new processes in stellar structure theory that go beyond the standard model framework. Of course we are aware of the crudeness of the present model: a more refined treatment would include the variation with depth of the horizontal turbulent diffusivity and of the Brunt-Väisälä frequency, as well as a more precise spin-down law, and it would not ignore the magnetic field.
Abdurashitov, J. N., et al. (SAGE Collaboration). 1996, Phys. Rev. Lett., 77,4708

Adelberger, E., et al. 1998, Rev. Mod. Phys., 70, 1265

Angulo, C. et al. 1999, Nucl. Phys. A, 655, 3

Alexander, D. R., \& Ferguson, J. W. 1994, ApJ, 437, 849

Bahcall, J. N., Krastev, P. I., \& Smirnov, A. Yu. 1998, preprint (hepth/9807216)

Baglin, A., \& Lebreton, Y. 1990, in IAU Colloq. 121, Inside the Sun, ed. G. Berthomieu \& M. Cribier (Dordrecht: Kluwer), 437

Balachandran, S. 1995, ApJ, 446, 203

Balachandran, S., \& Bell, R. A. 1998, Nature, 392, 791

Basu, S. 1997, MNRAS, 288, 572

Basu, S., \& Antia, H. M. 1995, MNRAS, 276, 1402

Burgers, J. M. 1969, Flow Equations for Composite Gases (New York: Academic)

Brun, A. S., Turck-Chièze, S., \& Morel, P. 1998, ApJ, 506, 913 (Paper I)

Brun, A. S., Turck-Chièze, S., \& Zahn, J. P. 1998, in Structure and Dynamics of the Interior of the Sun and Sun-like Stars, ed. S. G. Korzennik \& A. Wilson, (ESA SP-418; Noordwijk: ESA), 439

Cayrel, R. 1998, Space Sci. Rev., 84, 145

Caughlan, G. R., \& Fowler, W. A. 1988, At. Data Nucl. Data Tables, 40, 284

Chaboyer, B., \& Zahn, J. P. 1992, A\&A, 253, 173

Charbonneau, P., Christensen-Dalsgaard J., \& Henning, R. 1998, in IAU Symp. 181, Sounding Solar and Stellar Interiors, ed. J. Provost \& F. X. Smieder (Dordrecht: Kluwer), 161

Christensen-Dalsgaard, J. 1998, in Structure and Dynamics of the Interior of the Sun and Sun-like Stars, ed. S. G. Korzennik \& A. Wilson, (ESA SP-418; Noordwijk: ESA), 17

Choudhuri, A. M., Schüssler, M., \& Dikpati, M. 1997, A\&A, 319, 362

Cleveland, B. T., et al. 1998, ApJ, 496, 505

Corbard, T., Berthomieu, G., Provost, J., \& Morel, P. 1998, A\&A, 330, 1149

Corbard, T., Blanc-Féraud, L., Berthomieu, G., \& Provost, J. 1999, A\&A, 344,696

Donahue, R. A., Saar, S. H., \& Baliunas, S. L. 1996, ApJ, 466, 384

Dzitko, H., Turck-Chièze, S., Delbourgo-Salvador, P., \& Lagrange, G. 1995, ApJ, 447, 428

Elliott, J. R. 1997, A\&A, 327, 1222

Elliott, J. R., Gough, D. O., \& Sekii, T. 1998, in Structure and Dynamics of the Interior of the Sun and Sun-like Stars, ed. S. G. Korzennik \& A. Wilson, (ESA SP-418; Noordwijk: ESA), 763

Engstler et al. 1992, Phys. Rev. Lett. B, 279, 20

Fröhlich, C., et al. 1997, Sol. Phys., 170, 1

Gabriel, A. H., et al. 1997, Sol. Phys., 175, 207

Geiss, J., \& Gloeckler, G. 1998, Space Sci. Rev., 84, 239

Gonczi, G., Berthomieu, G., Corbard, T., Provost, J., Morel, P., \& the GOLF Team. 1998, in Structure and Dynamics of the Interior of the Sun and Sun-like Stars, ed. S. G. Korzennik \& A. Wilson, (ESA SP-418; Noordwijk: ESA), 461

Gough, D. O., \& Sekii, T. 1997, in IAU Symp. 181 Souding Solar and Stellar Interior, ed. J. Provost \& F. X. Schmieder (Dordrecht: Kluwer)

Grevesse, N., Noels, A., \& Sauval, A. J. 1996, in ASP Conf. Ser. 99, Cosmic Abundances, ed. S. S. Holt \& G. Sonneborn (San Francisco: ASP), 117

Grevesse, N., \& Sauval, A. J. 1998, Space Sci. Rev., 85, 161

Hampel, W., et al. (GALLEX Collaboration). 1996, Phys. Lett. B, 388, 384

Hata, N.. \& Langacker, P. 1997, Phys. Rev. D., 56, 6107
Iglesias, C., \& Rogers, F. J. 1996, ApJ, 464, 943

Kosovichev, A. G., et al. 1997, Sol. Phys., 170, 43

Lazrek, M., et al. 1997, Sol. Phys. 175, 227

Lebreton Y., Gomez, A. E., Mermilliod, J.-C., Perryman, M. A. C. 1997, in Proc. ESA Symp. Hipparcos: Venice '97 (ESA SP-402; Noordwijk: ESA), 231

Michaud, G., \& Proffitt, C. R. 1993, in IAU Colloq. 137, Inside the Stars, ed. A. Baglin \& W. W. Weiss (ASP Conf. Ser. 40; San Francisco: ASP), 246

Michaud, G., \& Zahn, J.-P. 1998, Theor. Comput. Fluid Dyn., 11, 183

Mitler, H. E. 1977, ApJ, 212, 513

Montalban, J., \& Schatzman, E. 1996, A\&A, 305, 513

Morel, P. 1997, A\&AS, 124, 597

Press, W. H. 1981, ApJ, 245, 286

Proffitt, C. R., \& Michaud, G. 1991, ApJ, 380, 238

Roca-Cortes, T., et al. 1998, in Structure and Dynamics of the Interior of the Sun and Sun-like Stars, ed. S. G. Korzennik \& A. Wilson (ESA SP-418; Noordwijk: ESA), 329

Rogers, F. J., Swenson, J., \& Iglesias, C. 1996, ApJ, 456, 902

Roxburgh, I. 1997, in SCORe'96, ed. F. P Pijpers, J. ChristensenDalsgaard, \& C. S. Rosenthal (Dordrecht: Kluwer), 23

Schatzman, E. 1993, A\&A, 279, 431

Schüssler, M. 1987, The Internal Solar Angular Velocity, ed. B. R. Durney \& S. Sofia (Dordrecht: Reidel)

Skumanich, A. 1972, ApJ, 171, 565

Soderblom, D. R., Pilachowski, C. A., Fedele, S. B., \& Jones, B. F. 1993a, AJ, 105, 2299

Soderblom, D. R., et al., 1993b, AJ, 106, 1059

Spiegel, E. A., \& Zahn, J. P. 1992, A\&A, 265, 106 (SZ92)

Suzuki, Y., et al. (SuperKamiokande Collaboration). 1998, in Neutrino 98 : Proc. XVIII Int. Conf. Neutrino Physics and Astrophysics, ed. Y. Suzuki \& Y. Totsuka (Amsterdam: North Holland)

Thompson, M. J., Toomre, J., \& the GONG Dynamics Inversion Team. 1996, Science, 272, 1300

Thorburn, J. A., Hobbs, L. H., Deliyannis, C. P., \& Pinsonneault, M. H. 1993, ApJ, 415, 150

Thuan, T. X., \& Izotov, Y. I. 1998, Space Sci. Rev., 84, 83

Tomczyk, S., Streander, K., Card, G., Elmore, D., Hull, H., \& Cacciani, A. 1995, Sol. Phys., 159, 1

Turcotte, S., et al. 1998, ApJ, 504, 539

Turck-Chièze, S. 1995, Adv. Space Res., 15, 85

. 1998, Space Sci. Rev., 85, 125 1999, NewA, 4, 325

Turck-Chièze, S., \& Basu, S. 1998, in Structure and Dynamics of the Interior of the Sun and Sun-like Stars, ed. S. G. Korzennik \& A. Wilson, (ESA SP-418; Noordwijk: ESA), 573

Turck-Chièze, S., et al. 1993, Phys. Rep., 230, 57

Turck-Chièze, S., et al. 1997, Sol. Phys., 175, 247

Turck-Chièze, S. et al. 1998, in Structure and Dynamics of the Interior of the Sun and Sun-like Stars, eds S. G. Korzennik \& A. Wilson, (ESA SP-418; Noordwijk: ESA), 555

Vauclair, S., \& Richard, O. 1998, in Structure and Dynamics of the Interior of the Sun and Sun-like Stars, ed. S. G. Korzennik \& A. Wilson (ESA SP-418; Noordwijk; ESA ), 427

Zahn, J. P. 1991, A\&A, 252, 179 1992, A\&A, 265, 115 . 1998, Space Sci. Rev., 85, 79 


\section{ERRATUM}

In the paper "Standard Solar Models in the Light of New Helioseismic Constraints. II. Mixing below the Convective Zone" by A. S. Brun, S. Turck-Chièze, and J. P. Zahn (525, 1032 [1999]), several corrections are required:

1. The words "greater than" just after equation (11) for the definition of $r_{b c z}$ should be removed.

2. The beginning of first sentence of the next paragraph should read: "With the latitudinal dependence of the angular velocity at the base of the convection zone borrowed from Thompson et al. (1996), $\Omega_{\mathrm{bcz}} / 2 \pi=456-72 x^{2}-42 x^{4} \mathrm{nHz}$," instead of " $\Omega_{\mathrm{bcz}}>/ 2 \pi=456-72 x^{2}-42 x^{4}$."

3. In the footnote to Table 1 , " $R_{\mathrm{bzc}}$ " should be " $R_{\mathrm{bcz}}$ " as it is appears for " $T_{\mathrm{bcz}}$ " in the same footnote.

4. In Table 2, in the "Parameters" column, " $\mathrm{i}_{0}$ " should be " $Z_{0}$," as in Table 1.

5. In Table 3, "Observaton" should be "Observation."

The Press sincerely regrets these errors. 